\title{
Sepsis Management with a Blood Purification Membrane: European Experience
}

\author{
Peter Pickkers ${ }^{a}$ Timon Vassiliou ${ }^{b}$ Valdis Liguts ${ }^{c}$ Federico Prato $^{d}$ \\ Pierre Tissieres $^{\mathrm{e}}$ Stephan Kloesel $^{\mathrm{f}}$ Franco Turani $^{\mathrm{g}}$ Dijana Popevski $^{\mathrm{h}}$ \\ Marcus Broman ${ }^{i}$ Ciprian Mihai Gindac ${ }^{j}$ Faouzi Saliba ${ }^{k}$ Marco Tengattini ${ }^{d}$ \\ Jacques Goldstein' Kai Harenski ${ }^{m}$
}

${ }^{a}$ Department of Intensive Care Medicine, Radboud University Medical Center Nijmegen, Nijmegen, The Netherlands; ${ }^{b}$ Department of Anaesthesiology and Critical Care Medicine, Academic Medical Centre, Klinikum

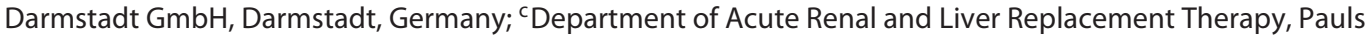

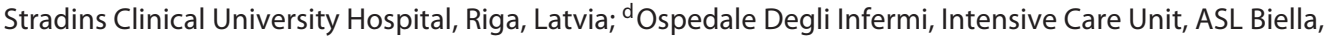

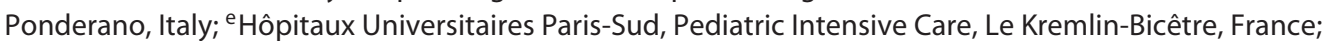
${ }^{f}$ Department of Anesthesiology and Intensive Care, GPR Klinikum Ruesselsheim, Ruesselsheim, Germany; ${ }^{9}$ Aurelia

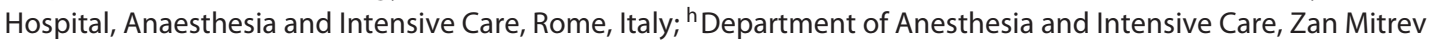
Clinic, Skopje, Republic of Macedonia; 'Perioperative and Intensive Care, Skåne University Hospital, Lund, Sweden; 'Department of Anaesthesia and Intensive Care, Emergency County Hospital "Pius Brinzeu", Timisoara, Romania; kHôpital Paul Brousse, Centre Hépato-Biliaire, Villejuif, France; 'Baxter World Trade SPRL, Acute Therapies Global, Braine-I'Alleud 1420, Belgium; 'm Baxter, Baxter Deutschland GmbH, Unterschleissheim, Germany

\section{Keywords}

Sepsis/septic shock · Acute kidney injury · Blood purification · Cytokines · Endotoxins

\begin{abstract}
Background: Septic shock is a leading cause of acute kidney injury (AKI). Endotoxins and cytokine levels are associated with the occurrence and severity of AKI, and different blood purification devices are available to remove them from circulation. One such device, oXiris, is a hollow-fibre purification filter that clears both endotoxins and cytokines. Due to limited evidence, clinical use of this device is not currently
\end{abstract}

advocated in guidelines. However, clinics do regularly use this device, and there is a critical need for guidance on the application of it in sepsis with and without AKI. Method: A modified Delphi-based method was used to collate European experts' views on the indication(s), initiation and discontinuation criteria and success measures for oXiris. Results: A panel of 14 participants was selected based on known clinical expertise in the areas of critical care and sepsis management, as well as their experience of using the oXiris blood purification device. The participants used different criteria to initiate treatment with oXiris in sepsis patients with and without AKI. Septic shock with AKI was the priority patient population, with oXiris used to rapidly improve haemody-

\section{KARGER}

E-Mail karger@karger.com www.karger.com/bpu (c) 2019 The Author(s)

Published by S. Karger AG, Basel

Karger

Open access

This article is licensed under the Creative Commons AttributionNonCommercial-NoDerivatives 4.0 International License (CC BYNC-ND) (http://www.karger.com/Services/OpenAccessLicense). Usage and distribution for commercial purposes as well as any distribution of modified material requires written permission.
Prof Dr. Peter Pickkers, MD, PhD

Department of Intensive Care Medicine (710)

Radboud University Medical Center Nijmegen

PO Box 9101, NL-6500 HB, Nijmegen (The Netherlands)

E-Mail Peter.Pickkers@ radboudumc.nl 
namic parameters. Achieving haemodynamic stability within $72 \mathrm{~h}$ was a key factor for determining treatment success. Conclusion: In the absence of established guidelines, users of hollow-fibre purification devices such as oXiris may benefit from standardised approaches to selecting patients and initiating and terminating treatment, as well as measuring success. Further evidence in the form of randomised clinical trials is urgently required.

(C) 2019 The Author(s)

Published by S. Karger AG, Basel

\section{Introduction}

Septic shock is a leading cause of acute kidney injury (AKI) among patients admitted to intensive care units (ICUs); it is associated with increased mortality and prolonged renal impairment in those who survive [1-3]. Observational studies of ICUs suggest that almost a quarter of patients are admitted with sepsis [2]. AKI is present in around half of those with severe sepsis $[3,4]$ and is significantly more frequent and more severe in patients presenting with sepsis on admission than in those without [2]. Endotoxins play a central role in the initiation and propagation of sepsis [5] by inducing the release of cytokines. Both endotoxins and cytokines may lead to the development of AKI $[6,7]$. Elevated circulating endotoxin concentrations relate to mortality [8]. Also, in patients with community-acquired pneumonia, those with increased interleukin- 6 and interleukin-10 levels were more likely to develop severe sepsis and had higher mortality than those who did not [9]. In addition, cytokine levels at ICU admission appear to relate to the severity of AKI [10]. The precise mechanisms of septic AKI are unknown but may involve endotoxin- and inflammation-mediated alterations in renal blood flow rate and distribution, as well as tubular damage due to the filtration of blood containing cytokines and other toxins [4]. Therefore, removal of endotoxins and cytokines may have benefits for patients with this condition [5], for example, potentially controlling haemodynamic instability.

Several blood purification devices that can remove both exogenous and endogenous inflammatory mediators are available [11]. Perfusion with a rolled woven-fibre polymyxin B haemoperfusion device (Toraymyxin, Toray Industries, Tokyo, Japan) selectively removes endotoxins from circulating blood in patients with sepsis or septic shock but does not capture endogenous inflammatory mediators [12]. The porous adsorbent polymer bead device (CytoSorb, CytoSorbents Corporation, NJ, USA) can be used in blood pump circuits to adsorb cytokines for conditions that involve excessive cytokine levels [13]; however, it does not capture endotoxins. Also, the hollow-fibre AN69 purification device (oXiris, Baxter, Meyzieu, France) has a large surface area with a dense membrane that facilitates the capture of both endotoxins and cytokines [14], as well as functioning as a renal replacement therapy (RRT) [14].

While there have been a handful of encouraging case reports [15-17], high-quality evidence is lacking concerning the effect of the oXiris device on clinical outcomes. Consequently, clinical use is currently not advocated [18]. The lack of established guidelines leads to variability in clinical practice, and it is presently unclear, for example, how users decide to initiate and terminate treatment or define treatment success. Knowing how experienced users think about these issues may have important implications for future trial design and, in due course, may streamline the current application of blood purification in patient care. To facilitate this process, we report the results of an Expert User Group Meeting on oXiris.

\section{Materials and Methods}

The Expert User Group Meeting was held in Frankfurt in September 2018 and was attended by 14 participants from 9 European countries. The meeting objectives were to better understand how oXiris is used in clinical practice, evaluate current prescription behaviours and gain insights into the current use of this purification device, that is, in what patient groups, when to start, when to stop, how to measure success. A modified Delphi-based method was used to collate the participants' views in 3 rounds [19]. Round 1 data were collected via an interactive PDF questionnaire. Round 2 was conducted at the face-to-face meeting; participants were divided into 4 subgroups and voted on questions posed by an independent facilitator. Round 3 was a second interactive PDF questionnaire designed to follow-up on discussion points raised at the face-to-face meeting. See the online supplementary material (for all online suppl. material, see www.karger.com/doi/10.1159/000499355) for further details.

\section{Results}

\section{Attendee Clinical Experience}

All participants $(n=14)$ worked in combined medical and surgical ICUs with a median number of 25 beds. Of these, 4 participants also reported working in a cardiac surgery ICU, one participant in a paediatric ICU and one participant in a hepatic ICU. 
Which Patient Group(s) Are Treated and Why?

The primary indication provided for using the oXiris hollow-fibre device was "sepsis"; however, within the group of sepsis patients, target groups varied and included "septic shock", "sepsis with AKI" and "sepsis without AKI". The criteria for the use of oXiris varied, although the presence of AKI was critical for the decision to initiate treatment (see below). The 2 main reasons for choosing the oXiris filter were "reduction of cytokines/endotoxins" and "improvement of haemodynamic instability". The perceived ease-of-use and filter characteristics were also considered beneficial.

\section{Which Criteria Are Used to Initiate Treatment?}

\section{Septic Shock Patients}

The effects of oXiris on supporting haemodynamic stability were most frequently mentioned by participants, thus advocating the use of the device in haemodynamically unstable patients. Haemodynamic parameters were judged as the critical criterion to initiate treatment. Also, elevated levels of inflammatory markers, AKI diagnosed per Kidney Disease Improving Global Outcomes guidelines and the presence of Gram-negative infection (as Gram-negative bacteria produce endotoxins) ranked highly (Fig. 1a). Nevertheless, it was also considered paramount that treatment with oXiris should be started as soon as possible. Therefore, not all participants would wait to reach an AKI diagnosis per the Kidney Disease Improving Global Outcomes criteria or for test results to become available; furthermore, in some situations, tests may not be practical or available (e.g., bedside endotoxin testing).

Furthermore, as most patients with septic shock are likely to be oliguric, urine output should be rapidly assessed along with haemodynamic criteria. However, the group members were divided on using oXiris if AKI was not present. No agreement was reached on the use or threshold levels of inflammatory markers, and it was recognised that cytokine measurements are not feasible in clinical practice at most institutes. There was consensus to treat sepsis patients if an infection was suspected but not proven, that is, before cultures were available. Apart from the unwanted delay in waiting for the cultures to become positive, it was recognised that apart from cytokines, circulating endotoxins are also found in patients with Gram-positive infections, presumably due to translocation of endotoxins from the gastrointestinal tract [20], so the use of oXiris in this group of patients is not contraindicated per se.

\section{AKI Patients}

Participants did not consider AKI in the absence of sepsis a sufficient indication to start treatment, as the oXiris filter was not considered to provide benefits over conventional RRT filters. Initiation criteria for the oXiris membrane for these patients are shown in Figure $1 \mathrm{~b}$. Participants were unanimous that all criteria must be associated with sepsis to consider using oXiris. Most participants would only use a hollow-fibre device to treat AKI if it was associated with septic shock, and most would use the mentioned criteria for treating sepsis itself.

Despite coagulation pathway activation often being a marker of sepsis [1], the group did not consider it appropriate to use a hollow-fibre device (instead of conventional RRT membranes) per se in patients with coagulatory dysfunction. While the oXiris filter is heparin coated, the device still requires management of anticoagulation, especially at lower blood flow rates [14], that is, the explicit and thorough treatment of sepsis with antibiotics and source control measures, combined with the use of anticoagulants [21]. As such, the group would recommend regional citrate anticoagulation (RCA), especially in patients with coagulation disorders.

Finally, they noted that while metabolic derangement is a marker of disease severity, it alone cannot be used as a criterion for initiating treatment with oXiris instead of a conventional membrane. There is no theoretical reason, and a lack of clinical evidence, to suggest that oXiris would confer additional benefits compared with conventional membranes in restoring metabolic derangements associated with AKI.
Fig. 1. Criteria for treatment initiation with oXiris in: (a) septic shock patients; (b) AKI patients; (c) trauma patients. Participants voted individually on the top criteria used to inform their respective decisions to initiate treatment with oXiris in each of the syndromes. Four subgroups then ranked each criterion, with the topranked criterion being given the highest score. These scores from the groups were consolidated and the criteria ranked by the sum of their scores, with the highest score being considered the most important by the group (left of the figure). Participants then voted individually to indicate whether they used the top criteria in their practice (right of the figure). SOFA, Sequential Organ Failure Assessment.
Pickkers et al. 


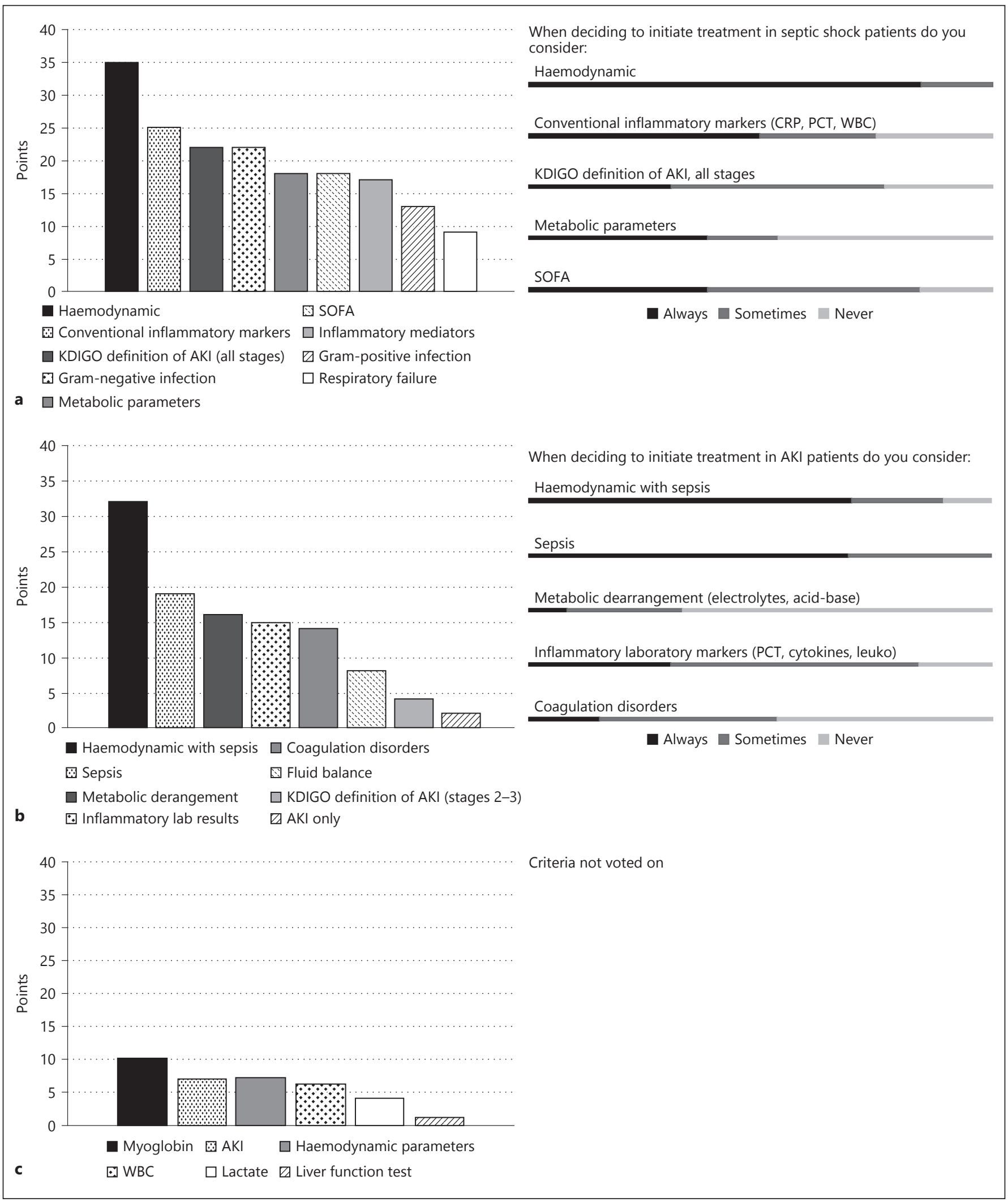


Trauma Patients

The group identified their criteria for treatment initiation in trauma patients (Fig. 1c). However, the consensus agreement was that the oXiris filter has no clearly indicated benefits over other filters in trauma patients with rhabdomyolysis and AKI. Therefore, the discussion was limited, and the criteria were not voted on.

\section{Other Patients}

The consensus agreement was that there are no other specific scenarios where oXiris may be useful, and discussion was limited. However, individual users reported using oXiris, in some cases as a preventative measure against further deterioration, in patients (with or without AKI) with pancreatitis, liver failure or myoendocarditis, or those ineligible for RCA. Users also identified practical situations where the hollow-fibre device would be appropriate, for example, a conventional filter clotting quickly in a patient who is ineligible for RCA.

\section{How Is Treatment Success Defined?}

The group noted a paucity of data on the definition of treatment success with the hollow-fibre device. Based on their personal experience, treatment success was defined by most participants as a rapid improvement in haemodynamic stability leading to a $50-75 \%$ reduction in vasopressor dose, generally within $24 \mathrm{~h}$ (online suppl. Fig. 2). For some, an improvement of haemodynamic instability should occur within $6 \mathrm{~h}$, while others appreciated improvements occurring up to $72 \mathrm{~h}$ following treatment with oXiris. It was recognised that in untreated patients, norepinephrine requirements might also decline. Therefore, in the absence of a randomised trial, the effect of the oXiris filter on haemodynamic instability cannot be claimed. While most participants would expect clinical improvement within $24-28 \mathrm{~h}$, duration of treatment and the number of filters used should not be fixed, but rather be dependent on efficacy. If the patient is no longer vasopressor dependent (within 24-72 h), treatment was considered successful and could be stopped.

\section{How Is Treatment Failure Defined?}

In addition to a worsening of the septic shock state as measured by conventional ICU scores, most of the group considered treatment unsuccessful if haemodynamic parameters had not improved within $72 \mathrm{~h}$. However, opinion was divided on the definition of haemodynamic stability: some considered an $80 \%$ reduction of vasopressor support in 48-72 h sufficient, while others suggested that incomplete removal of vasopressor support would define treatment failure. Consequently, treatment with the filter should not be continued due to futility in patients who remain vasopressor dependent for $>72 \mathrm{~h}$.

\section{Which Criteria Are Used to Discontinue Treatment?}

The group noted that treatment with the filter can be stopped because of success (treatment completed) or futility (treatment aborted). In contrast to the design of previous trials with other blood purification filters, it was felt that the duration of treatment with the filter (and consequently the number of filters used) should not be fixed, but rather be dependent on the efficacy, as described above.

Ending treatment with the oXiris filter due to success or futility is mainly based on the need for vasopressor treatment over time. Thus, if the vasopressor infusion is terminated, treatment with the oXiris membrane could be terminated too. Naturally, in patients with AKI in need of RRT, the continuous use of conventional membranes would still be needed. The dose of norepinephrine needed to maintain blood pressure was judged to be the most important discontinuation criterion (online suppl. Fig. 1), followed by the duration of use, with dose reductions of $50-75$ or $\geq 75 \%$ within $24 \mathrm{~h}$ being an indication of successful treatment (online suppl. Fig. 2). Further, early responses in terms of vasopressor dose reduction, usually observed within the first $12 \mathrm{~h}$, with clinical improvement in the first $24 \mathrm{~h}$ were used to define early improvement (online suppl. Fig. 2). With complete attenuation of the inflammatory response, typically within $24-48 \mathrm{~h}$, ranked highly as an outcome to determine the success of treatment (Fig. 2). However, while this endpoint may be specifically useful to compare groups in RCTs, determining the decrease in inflammatory mediators may be less useful when evaluating treatment success in an individual patient. Additional criteria were also discussed; Figure 2 and online supplementary Figure 2 for further details.

\section{What Are the Future Needs Related to the Use of a}

Blood Purification Membrane in Sepsis Patients?

The group identified a strong need for further evidence to facilitate clinical decision making and identify the specific patient populations that might benefit most. The group noted the need for RCTs comparing the efficacy of devices in patients with septic shock. Observational registry studies may be helpful to some extent, but the interpretation of any data may be hampered due to patient and treatment heterogeneity and the high risk of bias inherent to this type of research.
Pickkers et al. 


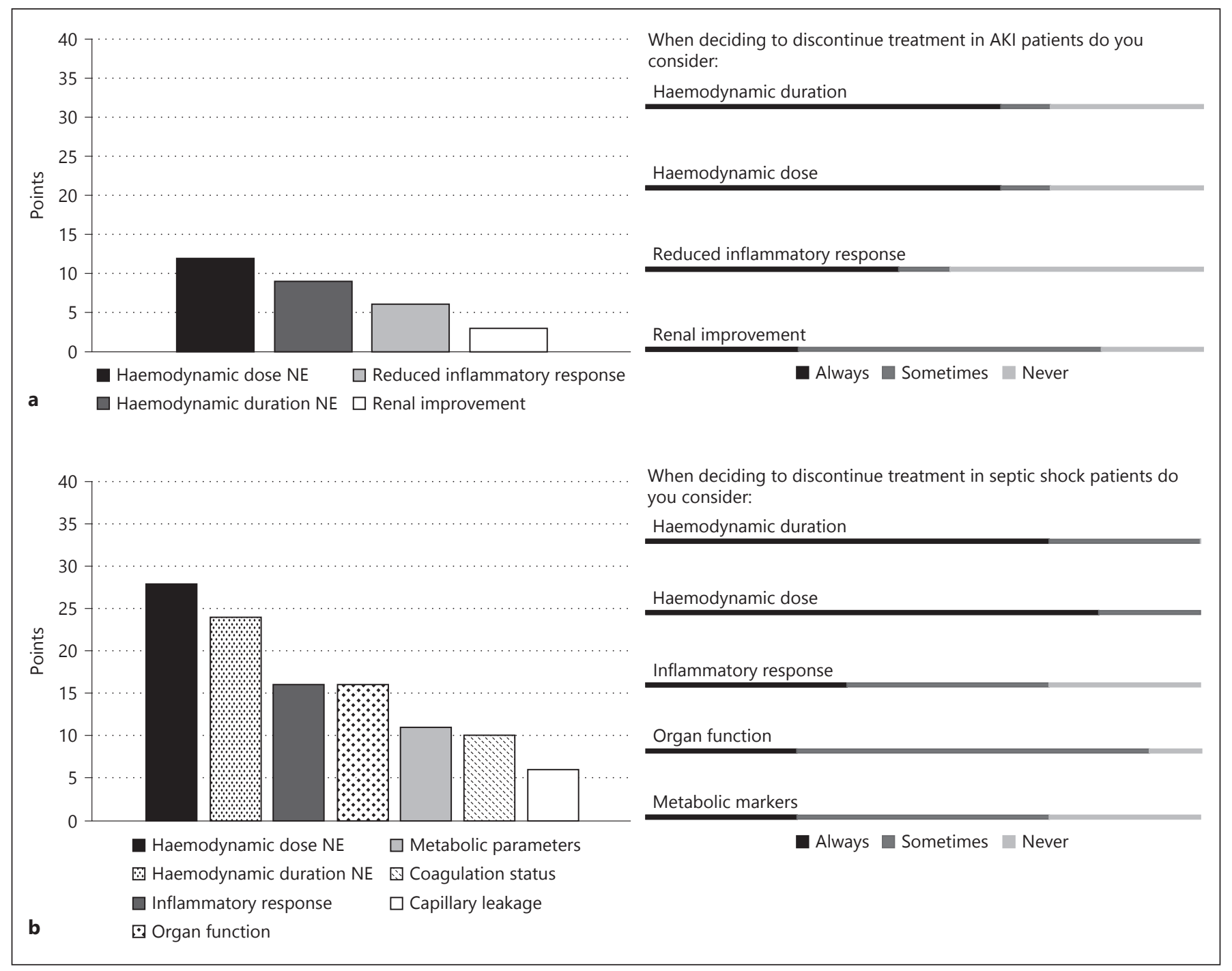

Fig. 2. Criteria for treatment discontinuation in: (a) AKI patients; (b) septic shock patients. Participants voted individually on the top criteria used to inform their respective decisions to discontinue treatment with oXiris in each of the syndromes. Four subgroups then ranked each criterion, with the top-ranked criterion being given the highest score. These scores from the groups were con-

The group did not consider mortality an appropriate primary endpoint per se, instead favouring norepinephrine dose reduction ( $>80 \%$; norepinephrine reduction within first $72 \mathrm{~h}$ ); positive haemodynamic response ( $>50 \%$ decrease at $72 \mathrm{~h}$ ); time to recovery from septic shock; RRT-free days; mechanical ventilation-free days; length of ICU stay; or change in Sequential Organ Failure Assessment (SOFA) score over time. Longer term effects, including renal recovery/chronic renal failure after ICU stay, were also mentioned.

Sepsis Management with a Blood Purification Membrane solidated and the criteria ranked by the sum of their scores, with the highest score being considered the most important by the group (left of the figure). The participants then voted individually to indicate whether they used the top criteria in their practice (right of the figure).

\section{Discussion}

During a typical Delphi process, $100 \%$ agreement is rare, and any consensus is the result of multiple rounds of voting and discussion that lead to a convergence of opinion. However, in areas where clinical evidence is limited, using a modified Delphi method may offer insight into the current practice of experienced users, so may provide guidance for current clinical application and future trial design. We identified commonalities as 
well as differences in how oXiris is used to manage patients in the ICU. Overall, the group agreed that the focus should be on the presence of sepsis as the treatment initiation criterion. There was consensus on the need to rapidly improve haemodynamic parameters being the critical factor in initiating treatment and defining treatment success. However, practices differed on when treatment was discontinued. These observations highlight a need for further guidance on this critical topic.

Each of the several devices designed to remove endotoxins or cytokines in patients with septic shock has different features and removal capabilities [11], which could enable treatment to be tailored to each patient's clinical needs. While acknowledging the benefits of a single device that can remove both endotoxins and cytokines, the participants did not advocate a broad clinical application of the hollow-fibre filter given the current lack of evidence of beneficial effects on clinical outcomes in critically ill sepsis patients.

The Acute Dialysis Quality Initiative Bogotá consensus concluded that blood purification might offer potential advantages over alternative therapies for sepsis [22]. However, the Acute Dialysis Quality Initiative acknowledges that slow progress has been made, in part due to a failure to phenotype patients in clinical trials [22]. While there are a number of marketed blood purification devices in use, due to a lack of high-quality evidence, the 2016 update to the Surviving Sepsis Campaign's International Guidelines for Management of Sepsis and Septic Shock makes no recommendation regarding their use [18]. In the meantime, results from RCTs using the Toraymyxin and CytoSorb devices have become available $[23,24]$. Unfortunately, in these studies, the devices did not appear to improve clinical patient-centred parameters [23, 24]. Furthermore, these studies did not separately evaluate septic patients with and without AKI; as such, any impact on pro-inflammatory response through the filter managing AKI is unknown.

The participants use the hollow-fibre device to treat sepsis or syndromes associated with sepsis. However, opinion was split over the treatment of sepsis in patients without AKI. The potential ability of oXiris to control immunodepression and inflammation by capturing endotoxins and cytokines and for treating suspected Gramnegative infections was recognised by the participants, although they acknowledged that currently there is limited information published covering clinical experience with oXiris [15-17].
The critical need to rapidly improve haemodynamic parameters in patients with septic shock is the most important reason to initiate treatment. The participants treat septic shock immediately and urgently in line with guidelines [18]; for them, this includes the use of the oXiris filter. For many septic shock treatments, prompt treatment may be crucial [25]. As such, while the participants identified other initiation criteria, it was also noted that it could be detrimental to the patient to await further test results before initiating treatment; indeed, optimal timing of treatment with oXiris needs to be established.

Effects of the use of oXiris in septic shock on endotoxin activity, SOFA score and procalcitonin levels have been reported $[15,17]$. However, while the participants acknowledge other parameters are important, only with the achievement of haemodynamic stability will treatment be considered clinically successful and can be discontinued, usually within $72 \mathrm{~h}$ of treatment. Should haemodynamic stability not be achieved within $24-72 \mathrm{~h}$, treatment would be considered unsuccessful and should be discontinued.

The participants were divided on whether the oXiris filter should be indicated in sepsis patients without AKI, and in relation to AKI and other applications of oXiris beyond syndromes associated with sepsis. Ultimately, further clinical evidence, most likely obtained through RCTs, is required to understand the optimal use of the hollow-fibre device and its impact on haemodynamic stability and SOFA scores.

\section{Limitations}

The participants were drawn from clinics across Europe only, and practices may vary in other regions, which could limit how transferable these discussions are. Furthermore, participants had clinical experience of using oXiris, which may have led to bias in the responses. The opinions reported here demonstrate the variance in managing sepsis across Europe within the group of oXiris users. Consequently, the outputs reported here should be used as suggestions to support practice, not as clinical guidelines, if clinicians are considering the use of the filter in their own clinical context.

\section{Conclusions}

The participants used different criteria to initiate treatment with oXiris in sepsis patients with and without AKI; however, there was a consensus that septic shock with AKI was the priority patient population. There is a press-
Pickkers et al. 
ing need to rapidly improve haemodynamic parameters in patients with septic shock, and achieving haemodynamic stability within $72 \mathrm{~h}$ is a key factor when determining treatment success. The ability of oXiris to remove endotoxins and cytokines in vitro is recognised as valuable, although there is still a need to understand these capabilities in the clinical setting. However, noting the absence of established guidelines on the use of hollow-fibre purification devices such as oXiris to treat sepsis or syndromes associated with sepsis, the group highlighted an urgent need for further evidence in the form of RCTs and/ or high-quality prospective studies to help guide decision making.

\section{Acknowledgements}

The authors acknowledge the insightful contributions of Xosé Pérez-Fernández.

Supported by Baxter Inc. Medical writing support was provided by Daniel Johnson, PhD, of SciMentum Inc. (Nucleus Global), funded by Baxter Inc., under the authors' conceptual direction and based on feedback from the authors. Peter Pickkers co-drafted the MS, and all authors reviewed and approved the final MS.

\section{Statement of Ethics}

The authors have no ethical conflicts to disclose.

\section{Disclosure Statement}

For all authors, attendance at the meeting was supported by Baxter Inc.

T.V. has received speaker fees for his scientific contributions at medical congresses and continuous RRT workshops from Baxter Germany Inc. T.V. has received honoraria for his contributions in the drafting of a handbook for continuous RRT from Baxter Germany Inc. P.P. has received speaker fees and travel reimbursements from Baxter Germany Inc. J.G. and K.H. are Baxter employees and hold Baxter stocks.

The other authors have no relevant COIs to disclose.

\section{Funding Sources}

Supported by Baxter Inc.

\section{Author Contributions}

All authors contributed to the concept, development and reviewing of all stages of this manuscript.

\section{References}

1 Zarjou A, Agarwal A. Sepsis and acute kidney injury. J Am Soc Nephrol. 2011 Jun;22(6): 999-1006.

2 Peters E, Antonelli M, Wittebole X, Nanchal R, François B, Sakr Y, et al. A worldwide multicentre evaluation of the influence of deterioration or improvement of acute kidney injury on clinical outcome in critically ill patients with and without sepsis at ICU admission: results from The Intensive Care Over Nations audit. Crit Care. 2018 Aug;22(1):188.

3 Poukkanen M, Vaara ST, Pettilä V, Kaukonen $\mathrm{KM}$, Korhonen AM, Hovilehto S, et al.; FINNAKI study group. Acute kidney injury in patients with severe sepsis in Finnish Intensive Care Units. Acta Anaesthesiol Scand. 2013 Aug;57(7):863-72.

4 Bellomo R, Kellum JA, Ronco C, Wald R, Martensson J, Maiden M, et al. Acute kidney injury in sepsis. Intensive Care Med. 2017 Jun;43(6):816-28.

5 Marshall JC. Endotoxin in the pathogenesis of sepsis. Contrib Nephrol. 2010;167:1-13.

6 Mårtensson J, Martling CR, Bell M. Novel biomarkers of acute kidney injury and failure: clinical applicability. Br J Anaesth. 2012 Dec; 109(6):843-50.

7 Gomez H, Ince C, De Backer D, Pickkers P, Payen D, Hotchkiss J, et al. A unified theory of sepsis-induced acute kidney injury: inflam- mation, microcirculatory dysfunction, bioenergetics, and the tubular cell adaptation to injury. Shock. 2014 Jan;41(1):3-11.

8 Opal SM, Scannon PJ, Vincent JL, White M, Carroll SF, Palardy JE, et al. Relationship between plasma levels of lipopolysaccharide (LPS) and LPS-binding protein in patients with severe sepsis and septic shock. J Infect Dis. 1999 Nov; 180(5): 1584-9.

9 Kellum JA, Kong L, Fink MP, Weissfeld LA, Yealy DM, Pinsky MR, et al.; GenIMS Investigators. Understanding the inflammatory cytokine response in pneumonia and sepsis: results of the Genetic and Inflammatory Markers of Sepsis (GenIMS) Study. Arch Intern Med. 2007 Aug;167(15):1655-63.

10 Payen D, Lukaszewicz AC, Legrand M, Gayat E, Faivre V, Megarbane B, et al. A multicentre study of acute kidney injury in severe sepsis and septic shock: association with inflammatory phenotype and HLA genotype. PLoS One. 2012;7(6):e35838.

11 Malard B, Lambert C, Kellum JA. In vitro comparison of the adsorption of inflammatory mediators by blood purification devices. Intensive Care Med Exp. 2018 May;6(1): 12.

12 Toray Industries. Data on file. Toraymyxin PMX-20R extracorporeal hemoperfusion cartridge. Instructions for use.
13 Cytosorbents Inc [Internet]. CytoSorb $300 \mathrm{~mL}$ Device-Instructions For Use. 2012. [cited 2017 Oct]. Available from: http://www.cytosorbregistry.org/wp-content/uploads/2014/10/2 CytoSorb_DeviceInstructionsForUse.pdf

14 Baxter. Data on file. oXiris. Instructions for use. 2017.

15 Shum HP, Chan KC, Kwan MC, Yan WW Application of endotoxin and cytokine adsorption haemofilter in septic acute kidney injury due to Gram-negative bacterial infection. Hong Kong Med J. 2013 Dec;19(6):491-7.

16 Turani F, Candidi F, Barchetta R, Grilli E, Belli A, Papi E, et al. Continuous renal replacement therapy with the adsorbent membrane oXiris in septic patients: a clinical experience. Crit Care. 2013;17(Suppl 2):P63.

17 Adamik BA, Smlechowicz JS, Zielinski SZ, Kübler AK. Use of extracorporeal elimination therapy for septic shock. Crit Care. 2013; 17(Suppl 2):P66.

18 Rhodes A, Evans LE, Alhazzani W, Levy MM, Antonelli M, Ferrer R, et al. Surviving Sepsis Campaign: International Guidelines for Management of Sepsis and Septic Shock: 2016. Intensive Care Med. 2017 Mar;43(3): 304-77.

19 Dalkey N, Helmer O. An Experimental Application of the Delphi Method to the Use of Experts. Manage Sci. 1963;9(3):458-67. 
20 Marshall JC, Walker PM, Foster DM, Harris D, Ribeiro M, Paice J, et al. Measurement of endotoxin activity in critically ill patients using whole blood neutrophil dependent chemiluminescence. Crit Care. 2002 Aug;6(4): $342-8$.

21 Levi M, van der Poll T. Coagulation and sepsis. Thromb Res. 2017 Jan; 149:38-44.

22 Kellum JA, Gómez H, Gómez A, Murray P, Ronco C; ADQI XIV Workgroup. Acute Dialysis Quality Initiative (ADQI) XIV Sepsis
Phenotypes and Targets for Blood Purification in Sepsis: the Bogotá Consensus. Shock. 2016 Mar;45(3):242-8.

23 Dellinger RP, Bagshaw SM, Antonelli M, Foster DM, Klein DJ, Marshall JC, et al.; EUPHRATES Trial Investigators. Effect of Targeted Polymyxin B Hemoperfusion on 28Day Mortality in Patients With Septic Shock and Elevated Endotoxin Level: The EUPHRATES Randomized Clinical Trial. JAMA. 2018 Oct;320(14):1455-63.
24 Schädler D, Pausch C, Heise D, Meier-Hellmann A, Brederlau J, Weiler N, et al. The effect of a novel extracorporeal cytokine hemoadsorption device on IL-6 elimination in septic patients: A randomized controlled trial. PLoS One. 2017 Oct;12(10):e0187015.

25 Seymour CW, Gesten F, Prescott HC, Friedrich ME, Iwashyna TJ, Phillips GS, et al. Time to Treatment and Mortality during Mandated Emergency Care for Sepsis. N Engl J Med. 2017 Jun;376(23):2235-44. 